\title{
Helping Cancer Survivors Return to Work
}

\author{
Presented by Amye J. Tevaarwerk, MD
}

\begin{abstract}
Work limitations due to health problems can range from mild or transient limitations to persistent, long-term dysfunction and can lead to employment instability, underemployment, and even loss of employment. In fact, compared with a healthy matched control population, cancer survivors are 1.37 times more likely to be unemployed. Because patients with metastatic disease are particularly vulnerable, proactive discussion regarding the potential impact of treatment on employment and work outcomes may be beneficial. However, employment and financial toxicity are not topics that clinicians are necessarily trained to address. Financial counselors or patient navigators may be better able to offer a personalized approach and help survivors navigate the complex resources that are involved. Additional research into cancer-related work outcomes is needed.
\end{abstract}

J Natl Compr Canc Netw 2021;19(5.5):662-664 doi: $10.6004 /$ jnccn.2021.5002

As many as $46 \%$ of people diagnosed with cancer are between the ages of 20 and 64 years, ${ }^{1}$ which is classically defined as the working age population in the United States. However, according to Amye J. Tevaarwerk, MD, Associate Professor, University of Wisconsin School of Medicine and Public Health, and Carbone Cancer Center, it's unclear what fraction of the cancer survivor population is actually working at the time of diagnosis versus in school versus retired. It's also unclear what percentage of this population is being or has been treated with curative or palliative intent.

During the NCCN 2021 Virtual Annual Conference, Dr. Tevaarwerk discussed the impact that cancer and its treatment can have on a survivor's work status, work performance, and job satisfaction. Dr. Tevaarwerk also reviewed interventions that may enhance return to work in cancer survivors.

\section{Terms and Definitions}

Although frequently used, the phrase "return to work" may be a misnomer because it implies that cancer is a linear journey that moves from screening to diagnosis to active treatment into survivorship and maintenance care. In reality, however, cancer journeys are not always so straightforward.

"For patients with metastatic disease, the active treatment phase may never really end. Then, there are individuals who experience a recurrence and must circle back to the active treatment phase," said Dr. Tevaarwerk. "The phrase 'return to work' implies that a cancer survivor stops working and then restarts, only once. The truth is that patients with cancer may not ever stop working, or they may stop and start more than once, or they may stop and restart at a very different level or job."
Dr. Tevaarwerk noted the following useful definitions to consider:

- Individuals are considered employed if they perform any work at all for pay or profit.

- Work limitations are changes in the ability to work or in productivity at work and include physical limitations, mental/emotional limitations, or both.

- Limitations due to health problems can range from mild or transient limitations (eg, a brief 4-week surgical recovery before returning to an office job) to persistent, long-term dysfunction (eg, persistent neuropathy impacting the ability to manipulate tools at work).

\section{What Are the Consequences?}

These limitations can lead to a variety of outcomes, including increased struggle but no decrease in productivity, employment instability (eg, change in job or employer), underemployment (ie, accepting less demanding or part-time positions), or even nonemployment (ie, early retirement or loss of employment).

Less work and decreased income can also exacerbate financial toxicity, which in turn might lead to treatment delays, lack of treatment adherence, and distress. Furthermore, said Dr. Tevaarwerk, work is more than income. Survivors need to work for insurance and income purposes, but work also conveys a sense of normalcy and may be an important source of social contact. "Physical, emotional, and functional well-being and cancer-specific symptoms significantly predict presenteeism," she said. "Conversely, higher activity impairment and overall work impairment are significantly associated with poorer health-related quality of life." 
One study showed that cancer survivors who were employed 5 years after diagnosis had better quality-oflife outcomes compared with those who were unemployed. ${ }^{2}$ Caution should be used in interpreting these findings - after all, many factors may impact the decision to continue working. However, statistics show that cancer survivors are 1.37 times more likely to be unemployed than a healthy matched control population. ${ }^{2}$

\section{Work-Related Productivity}

Work-related productivity loss due to health may change significantly, with lower levels of productivity occurring after diagnosis and at the end of treatment, for those treated with curative intent. However, the degree of impairment may vary according to tumor site and country of treatment. The main driver of nonemployment appears to be related to absenteeism. "Loss of working days due to cancer treatment is an important reason for impaired productivity," said Dr. Tevaarwerk. "After the patient is off treatment, absenteeism my decrease, of course, if they are being treated with curative intent."

A study by Tevaarwerk et $\mathrm{al}^{3}$ of 120 patients receiving chemotherapy with curative intent who were employed at diagnosis and intending to work or return to work highlights a number of potential risk factors. "Return to work for patients with cancer is very complicated and not just limited to disease status and symptom burden," she said. "You may need to consider how such factors interact with survivor characteristics such as age, sex, race, education, and marital status, and how these interplay with job type or flexibility, as well as work environment."

Patients with metastatic disease may be especially vulnerable. According to Dr. Tevaarwerk, metastatic cancer can affect physical and mental ability to work, the ability to complete work in a timely manner, and relationships with employers and coworkers. Results from the ECOG-ACRIN Symptom Outcomes and Practice Patterns study, ${ }^{4}$ which investigated factors associated with the employment of 680 patients with metastatic cancer, found that $45 \%$ had stopped working due to illness. "That's a fairly profound impact on work outcomes, at least as defined by employment, for patients with metastatic disease."

\section{Interventions}

For patients with metastatic disease in the curative-intent setting, proactive discussion regarding the potential impact of treatment on employment and work outcomes might be beneficial. According to Dr. Tevaarwerk, survivors desire guidance and support from clinicians on the topic. 5 When discussing how treatment may impact work outcomes, however, it can be difficult if there are no good alternative therapy options to offer patients.

"Sometimes, in the curative-intent setting, providers don't have the luxury of alternative treatment options, but shared-decision making could mitigate the impact," she said. "In the metastatic setting, however, recognition of patient priorities considering treatment and contextual characteristics, such as efficacy, toxicities, impact on daily life, and responsibilities, are essential for autonomy in the decision-making process." That being said, Dr. Tevaarwerk acknowledged that employment and financial toxicity are not topics that clinicians are necessarily trained to address. Financial counselors or patient navigators, on the other hand, may be better able to offer a personalized approach and help survivors navigate the complex resources that are involved. Although not every center will have these resources, she said, navigators or social workers can help assess financial distress with patient-centered instruments to measure financial toxicity, provide appropriate counseling, address social determinants of health and underserved groups, and mobilize resources available in the community. "Nevertheless, it is important for clinicians to help by addressing these concerns and discussing the impact on work in the context of treatment decisions," she said.

When it comes to lifestyle and work, there is also circumstantial evidence regarding body weight. Dr. Tevaarwerk reported that excess body weight at breast cancer diagnosis is independently associated with increased rates of non-return to work 2 years after diagnosis among young, previously employed breast cancer survivors. " "There's a lot of ongoing research, but there's at least anecdotal evidence that body weight may impact symptoms," she said. "Although many of these symptoms have evidenced-based interventions, we haven't really studied whether intervening on those symptoms really impacts work outcomes."

According to Dr. Tevaarwerk, the NCI identified several areas for continued research in a 2018 paper. $^{8}$ As a start, employment outcomes should be included in cohort studies of cancer survivors to characterize factors impacting cancer-related work outcomes. Longitudinal studies should also evaluate facilitators and barriers to employment over time and identify modifiable risk factors for different employment outcomes. In addition, future studies should test multidisciplinary rehabilitation interventions in the United States using larger samples and experimental designs.

Finally, said Dr. Tevaarwerk, strategies should be tested to help cancer survivors and their families understand their legal rights pertaining to leave, job protection, and insurance coverage and to facilitate their access to state, municipal, and community-level programs and policies that are relevant for their needs.

Disclosures: Dr. Tevaarwerk has disclosed no relevant financial relationships.

Correspondence: Amye J. Tevaarwerk, MD, University of Wisconsin, 1111 Highland Avenue, WIMR 6037, Madison, WI 53705.

Email: at4@medicine.wisc.edu 


\section{References}

1. National Cancer Institute, National Institutes of Health, Division of Cancer Control \& Population Sciences. Employment outcomes among cancer survivors. Last updated: May 16, 2019. Accessed February 1, 2021. Available at: https://healthcaredelivery.cancer.gov/employment

2. de Boer AG, Taskila T, Ojajärvi A, et al. Cancer survivors and unemployment: a meta-analysis and meta-regression. JAMA 2009; 301:753-762.

3. Tevaarwerk AJ, Kwekkeboom K, Buhr KA, et al. Results from a prospective longitudinal survey of employment and work outcomes in newly diagnosed cancer patients during and after curative-intent chemotherapy: a Wisconsin Oncology Network study. Cancer 2021;127:801-808.

4. Tevaarwerk AJ, Lee JW, Terhaar A, et al. Working after a metastatic cancer diagnosis: factors affecting employment in the metastatic setting from
ECOG-ACRIN's Symptom Outcomes and Practice Patterns study. Cancer 2016;122:438-446.

5. Taskila T, Lindbohm ML, Martikainen R, et al. Cancer survivors' received and needed social support from their work place and the occupational health services. Support Cancer Care 2006;14:427-435.

6. Hoving JL, Broekhuizen MLA, Frings-Dresen MHW. Return to work of breast cancer survivors: a systematic review of intervention studies. BMC Cancer 2009;9:117.

7. Di Meglio A, Menvielle G, Dumas A, et al. Body weight and return to work among survivors of early-stage breast cancer. ESMO Open 2020;5:e000908.

8. de Moor JS, Alfano CM, Kent EE, et al. Recommendations for research and practice to improve work outcomes among cancer survivors. J Natl Cancer Inst 2018;110:1041-1047.

\title{
Explore Oncology From Every Angle
}

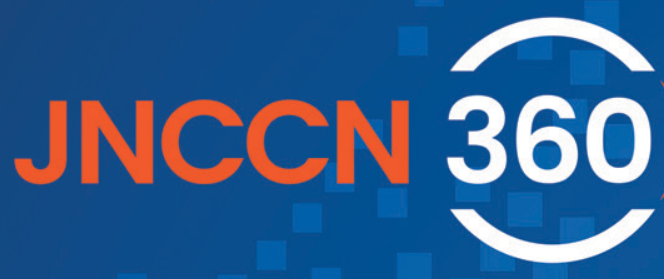

\author{
The Hub for Disease-Specific Clinical Information \\ JNCCN Spotlights: \\ $>$ Links to professional \\ exclusive commentary \\ and patient resources \\ about current therapies \\ > Video interviews with experts \\ - Summaries of news \\ and medical literature
}

\section{JNCCN360.org}

- AARBORSIDE

The nexus of knowledge
National Comprehensive NCCN Cancer 